\title{
Serdev Suture for Buttock’s Lift - Ambulatory Scarless Procedure
}

\author{
Nikolay P. Serdev ${ }^{*} \S$
}

Medical Centre "Aesthetic Surgery, Aesthetic Medicine", Sofia, Bulgaria

\begin{abstract}
Background: This article presents the author's technique and experience in the treatment of the flaccid "unhappy buttock" form with his surgical procedure of buttock lift by suture, without incision scars. The author first presented this new operation technique on a national level at the 2nd Annual Meeting of the National Bulgarian Society for Aesthetic Surgery and Aesthetic Medicine in Sofia on March 18, 1994 [1] and internationally at many scientific meetings over the world $[2,3,4 \ldots]$. The result is a visual change in the buttock position to a higher one, which elongates the lower limbs and changes the proportions between lower and upper half of the body.
\end{abstract}

Objective: The aim of this study is to describe a mini-invasive procedure of beautification of the buttock form without scars by creating a lifting effect on the buttock's subcutaneous tissue, using a suture that takes the inferiorly positioned deep fibrose tissue and fixes it upwards to the sacro-cutaneous fascia, discovered by the author. Aesthetic and technical considerations required properly sculpting the buttocks into a higher position, demonstrating nicely rounded form.

Methods: 1032 female patients, and 26 male patients aged 18-62 years, with ptosis and cellulite on the buttocks were treated since 1993 on an outpatient basis by the "Serdev suture technique without visible scars". Important instrumentarium is a long, curved, elastic needle and Polycon semi-elastic Bulgarian antimicrobial polycaproamide long term (in 2 years) absorbable surgical threads Polycon, produced in Bulgaria. This operation has been performed either alone or after ultrasonic assisted liposculpture (UAL) that reduces the amount of fat and heaviness.

Results: All patients reported a high degree of satisfaction. A stable improvement in the buttock position and form was observed for the period described. In the postoperative period the complication rate was minimal and resolved in the first 4-5 days post operative period. The skin puncture in the perianal zone makes antibiotic prophylaxis obligatory as well as a strict follow up for the first 7 days. Some pain in the sitting position was observed for at least 5 to 10 days, but all other social and professional duties and activities were possible.

Conclusions: This outpatient procedure is effective in the correction of buttock laxity and ptosis and creates a new form, universally accepted as "happy buttocks".

Keywords: Serdev Suture, Buttock's lift, Serdev needles, Semi-elastic Polycon thread.

\section{INTRODUCTION}

As more people seek body contour surgery, we should use our growing, developing knowledge and surgical experience to create new non-scaring surgical procedures for beautification, where formerly results of body contour surgery in areas like the buttocks have been less satisfying. A very small number of techniques are available for correction of the form and aesthetics of the buttocks, especially in cases of lax and ptotic buttocks. Non-scarring and sparing methods are preferred and requested by patients. So far, the minimally invasive technique most utilized for the fatty tissues has been liposuction exclusively. However the use of ultrasonic assisted liposculpture (U.A.L.) alone cannot lift or tighten up loose, lax and ptotic buttocks, and in our hands is combined with Serdev suture technique for buttock lift. In literature, liposuction is mostly followed by excision

*Address correspondence to this author at the Medical Center "Aesthetic Surgery and Aesthetic Medicine", 11, "20th April" St., 1606 Sofia, Bulgaria; Tel: +359 88571 9696; Fax: +359 2 9515668; E-mail: serdev@gmail.com

${ }^{\S}$ President, The Bulgarian Society of Aesthetic Surgery and Aesthetic Medicine. of tissue or other surgical methods. To improve the gluteal region in those patients whose problem is skin flabbiness rather than excessive fat, combinations of techniques are performed: liposuction, lipoinjections, implants for augmentation and lipectomy.

The aim of our paper is to present a new outpatient scarless buttock lift surgical procedure by closed approach suture that can meet patients requirements for beautification of buttocks form and position without scars, that has an uncomplicated, rapid post operative recovery period and is long lasting.

\section{Special Threads and Needles}

We present the Serdev ${ }^{R}$ needles and the semi-elastic longterm absorbable Bulgarian threads that has made possible the introduction of a successful scarless, closed approach technique of Serdev Suture ${ }^{\mathrm{R}}$ buttock lift with almost no downtime.

\section{Anatomy}

The well accepted gluteal position is the position of the m. gluteus maximus. The musculo-sceletal framework is 

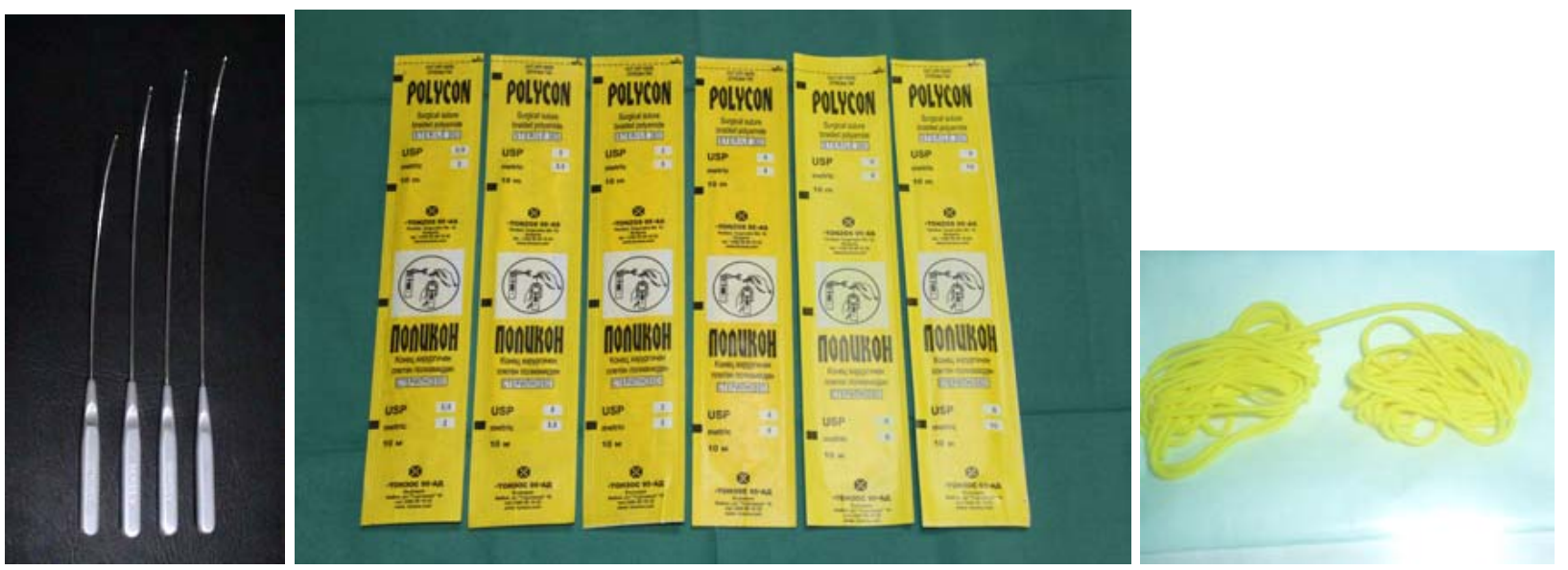

Fig. (1). Serdev ${ }^{\mathrm{R}}$ Needles and Bulgarian semi-elastic, anti-microbial, long term absorbable threads.

normally nicely formed. Unfortunately, female structure mostly includes an inferiorly positioned fatty tissue deposit, elongating ladies buttocks in lower aspect. These elongated hanging buttocks are visible from the frontal view as well that shortens the lengths of female lower limbs from the rear view. The hanging soft tissue is well known as "unhappy buttocks", different from the high gluteal position, called "happy buttocks".

The gluteal fatty tissue includes fibrotic fibres, fascial layers and trabecular system attaching the skin to the gluteus maximus fascia. The fascial tissue represents a flexible support for the soft framework of the human body. It forms a stable network for subdermal and deep fat layers, as well as cases for muscles, and sheaths for blood vessels and nerves. We use this stable fascial structure to fix higher the buttock soft tissue.

\section{Serdev Fascia}

Important anatomy landmark is the "Serdev fascia" or "sacro-cutaneous fascia" discovered by the author, fixing the skin to the lateral borders of the sacrum and the only safe fibrotic structure to hold the buttock's heaviness in the new position. It is perpendicular to skin and sacrum and is located in the line between the Venera dimples at the sacroiliac joint and the upper point of the inter-gluteal fold at the sacrocococcygeal joint.

\section{PATIENTS AND METHOD}

The primary indication for buttock lift surgery by suture is the moderate to severe soft-tissue laxity in the lower trunk with minimal or mild residual fat deposits. If significant fat deposits are presented, we initially treat patients with UAL to reduce the volume and heaviness of the buttock fat tissue [2].

In selected cases, UAL was performed for body beautification and sculpturing, and to reduce the buttocks volume and heaviness.

The Serdev suture ${ }^{R}$ was created for aesthetic purposes with the intention of creating a higher and more rounded attractive buttocks, at the same time creating a visible elongation of the legs and a change to the correlation of body to lower limbs length. True buttock sculpting demands a three-dimensional artistic understanding of the anatomic and surgical adipose layers of the central trunk, a stable immobile fixation to Serdev fascia and semi-elasticity of the suture to hold the mobile fibrotic buttocks tissue. This is essential in preventing complications from the buttock lifting such as trauma, inner tissue decubiti etc.

(A)

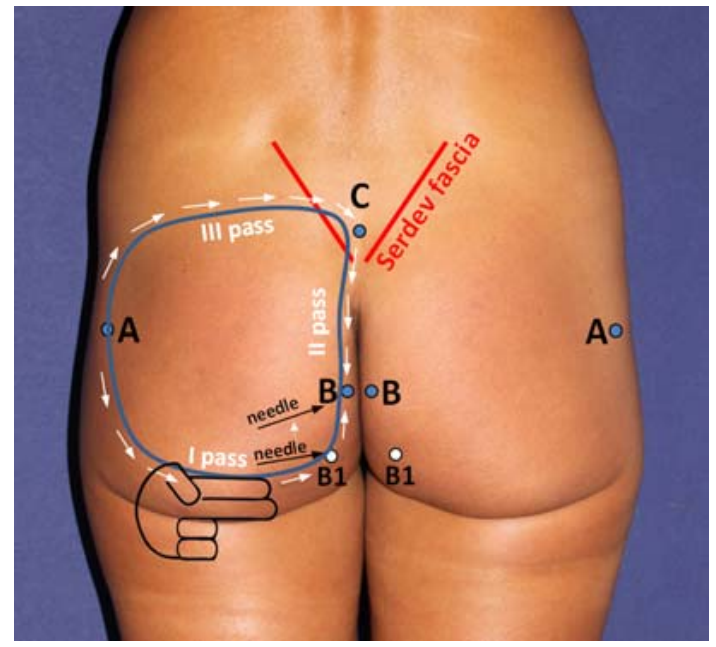

(B)

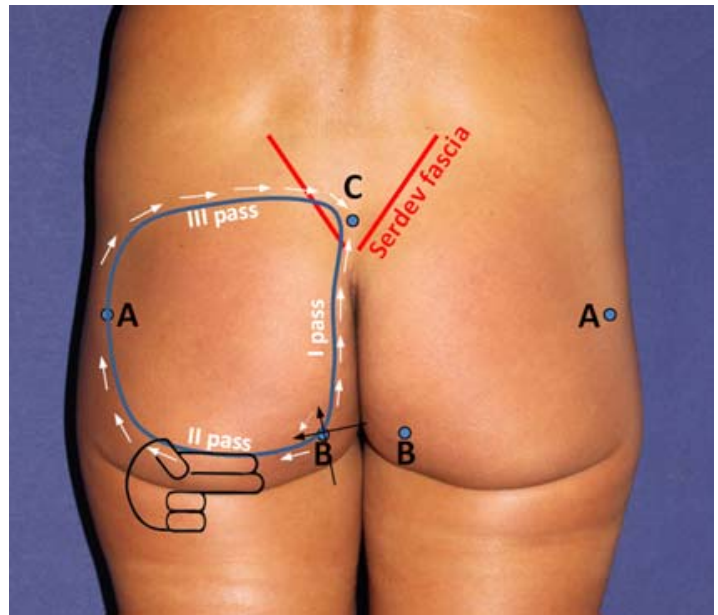

Fig. (2). A. Suture starting from lateral. B. Suture starting from medial. Arrows show needle direction. The Serdev sacro-cutaneous fascia perpendicular to sacrum and skin to hold the buttocks after the lift. 
Our surgical technique contains a fixation of the complete supeficial fascial system of the buttock's soft tissue (2 fingers higher than the infra-gluteal fold - Fig. 2) through a special long needle (Fig. 1) and a suture technique including 3 steps: The first step for fixation of the subdermal fascial tissue could begin on the lateral aspect of the buttock - Point A (Fig. 3) mostly using the same penetration point, like in liposuction, UAL or using an old one) and ends medial $2-3 \mathrm{~cm}$ higher and lateral from the anus at point B, while the second begins on the upper penetration point $C$ and ends on the lower aspect of the inter-gluteal fold at B. The third fixation passes convex the upper part of gluteus maximus and ends at $\mathrm{C}$, fulfilling the circular character of the suture. Elastic tightening of this suture assures a higher fixation of the gluteal fibrous layers of the lower part of the buttock to the Serdev sacro-cutaneous fascia. At the same time it corrects the trabecular system of the skin in a superficially convex "bucket". This superficial roundness is moved superiorly to its best position. The fixation of the suture to the stable inelastic Serdev fascia, maximally guaranties the longevity of the aesthetic effect. The elastic quality of the antimicrob polycaproamide Polycon that we use, reduces the possibility of decubiti of the fibrotic tissue and reduces complications.

(A)

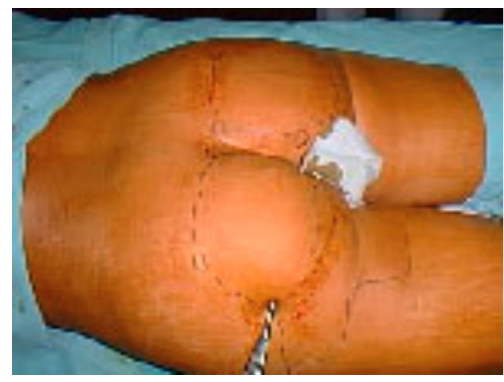

(B)

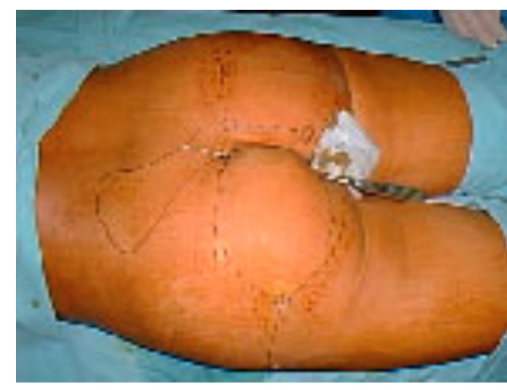

(C)

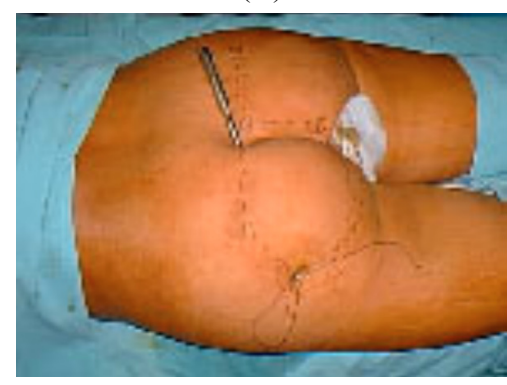

Fig. (3). A, B, C. Three steps minimum are necessary for a complete fixation of the buttock superficial fibrous system. It realizes a stable fixation of the lower buttock fibrotic soft tissue to the Serdev sacro-cutaneous fascia.
The 3 steps could be done in another order, starting from the medial lower aspect as shown on Fig. (3) without to exit at point $\mathrm{B}$ that is sometimes easier for beginners.

The buttock lift by suture requires 10 to $15 \mathrm{~min}$ of operating time per side, no blood transfusions, no stay at the clinic, no nursing care, and not more than a day or two off work.

More than thousand cases of laxity and ptosis in the gluteal area were operated during the years 1993-2012 to lift the buttocks for aesthetic reasons. This mini-invasive method, new in the beginning became fast a standard procedure internationally. It became very popular among patients and is accepted better than any excision surgery. Patients were informed about possible expected and unexpected risks of surgery and sign an informed consent. They were informed about the qualities of the Bulgarian semi-elastic antimicrobial thread, about the minimal trauma and the pain in sitting position in the first 3-6 days after the mini-invasive procedure, about the hygienic requirements and gradual exercise during the first month and a half after the procedure.

Positive fact is that the suture method for buttocks lift can be repeated and in 5 difficult cases of marked heaviness, and ptosis we have planed lifting in 2 steps. A second step could be planned at least after a year. In one patient we have done a second step after 19 months and a third step after another 18 months due to patients wish for beauty. We consider this case as an open door for suture lift repetition and possibility to maintain our patients in a good form with a short post operative downtime technique.

Our patients ranged in age from 18 to 62 years.

In the same session, patients who had moderate lower trunk and lower limb cellulite were treated with additional ultrasonic liposculpture of the lower body.

\section{RESULTS}

To judge a buttocks lift is difficult: UAL does not lift - it was used in our patients to reduce buttocks heaviness and to beautify the body shape around; A table is hard to be added because each patient's body changes much and differently during the years, some gain, other loose weight, body height reduces with time; both lower extremities and both gluteal folds are not equal; Sagging starts and is different in each patient and has no universal measure - but sagging after a suture lift starts from a higher (lifted) position; Gluteal fold is not exactly raised - the buttocks soft tissue is raised 2 fingers higher from the gluteal fold as drown in Fig. (2) to stretch and flatten a bit the fold; The suture material has a short elasticity to reduce trauma in fixation points, it is absorbable after final fibrosis1,5 - 2 years, because foreign bodies are unnecessary; The trauma of the blunt needle is minimal, the thread is antimicrobial, antibiotic for 7 days was administered; Follow up is strictly for the first 7 days post-op in the clinic, that reduces early post-op sequels; All these reduces risks; Effect is scarless and immediate and patients judge their satisfaction. Only complain was early post-op pain. We treat the early post-op possibilities for infection with daily showers, disinfection and change of bandages in the first seven days. Late post-op complains could appear as pain in a point after inadvisable exercising 
that appeared in 23 patients and was treated successfully in days with rest, repeated antibiotic for minimum 5 days and non-steroid anti-inflammatory suppositories.

The cosmetic results were evaluated with preoperative and postoperative photographs.

No patient was dissatisfied with the immediate and early result. Patients considered their results good, even in $21 \%$ excellent, during the first two years (Figs. 4-9).

407 patients were followed for more than 15 years.

After 2 years, 74 patients report loosing the effect, the rest of the patients consider that the aging process of the buttocks is delayed in comparison to other body parts, form is still better than before, and even if normal ptosis is presented the postoperative ptosis is developing from a higher tissue level.

After 10 years, 174 patients still see preserving of some change in the buttocks form and projection, and better proportions in comparison with the pre-operative status.

On the $15^{\text {th }}$ year, 138 patients still maintained their total body form and good shape and 76 of them were still satisfied with the buttock form, projection, and body proportions.

\section{RISKS AND COMPLICATIONS}

All surgical procedures are associated with risks. They may be divided into undesired sequelae, which are normal and expected, and complications, which are not normal or expected. The undesired sequelae of suction lipectomy are contour irregularities, hypaesthesia, oedema, ecchymosis, and pigmentary deposits. The potential complications are blood loss, haematoma, seroma, infection, greater saphenous vein thrombosis, fat emboli, and skin slough [3, 4]. In excision procedures additional complications are spreading scars as a result of tension, and occasional delayed healing of tense wounds. Excision buttock lifts has not gained widespread acceptance because of problems such as large trauma and blood loss, prolonged post op period, early inferior scar migration, and recurrence of ptosis. The most frequent complaint is unacceptable scarring and hypaesthesia [5, 6]. Lipoinjection complications are gluteal temporal hyperaemia and erythema, corresponding to fat necrosis [7-9].

Acceptance of bodily proportions in races different from those of Caucasians has to be foreseen. Furthermore, AfroAmericans and Japanese tend to hypertrophic scarring [10].

In our Patients we have not observed neurologic problems (ischiadic pain) because we do not touch the sacrococcygeal fascia, we use the Serdev fascia for suspension. Trauma is minimal and oedema is not visible for others. Ecchymosis are rarely presented.

We have had no thread expulsion. Sometimes we repeat the buttocks suture lift for beautification or in steps if too much heaviness and flabbiness are presented.

Infection: In our method we observed one case with a painful firmness around one of the entry points and another case with a local infection in one of the wounds. The cause for the first complication was the rigid nylon suture we have used in our first patient, causing a tissue decubitus in the point of tension on the soft tissue. After this complication
(A)

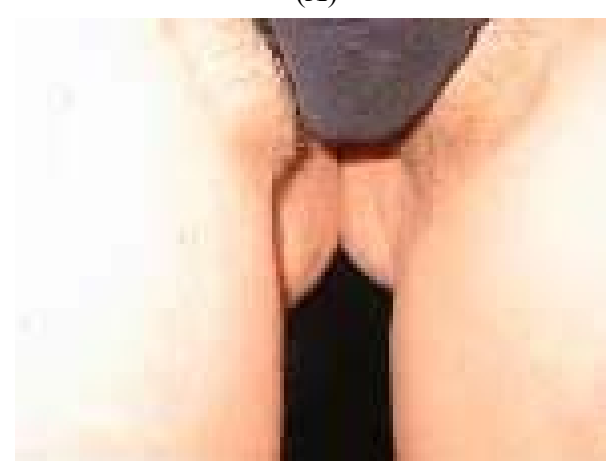

(B)

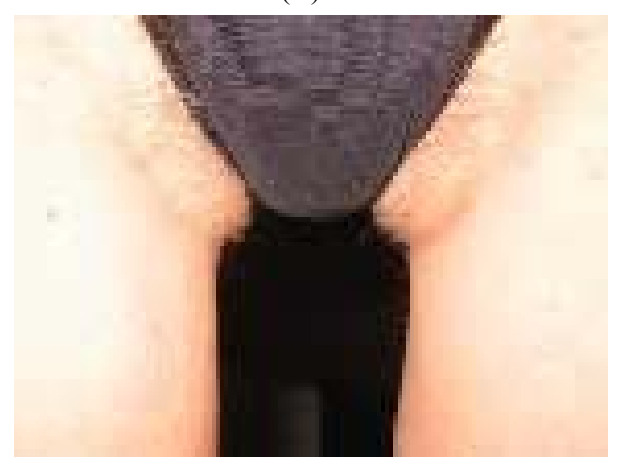

Fig. (4). A. Before. "Unhappy", sagging, loose buttock soft tissue, dropping between the thighs, often depresses ladies and they ask for buttock lift. B. After. "Happy" Buttocks after a suture lift. The idea to lift the hanging buttocks that optically elongates the legs is realized using the Serdev suture technique.

(A)

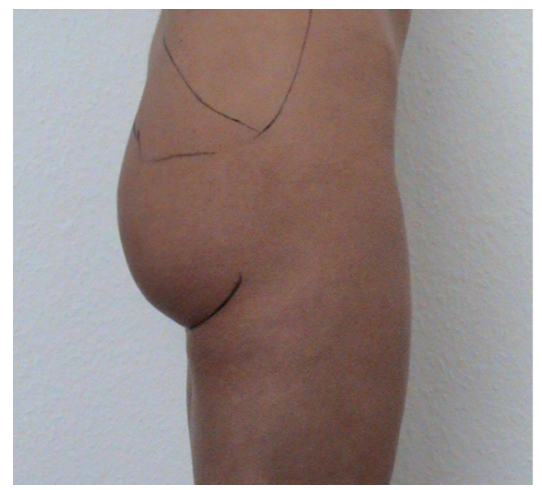

(B)

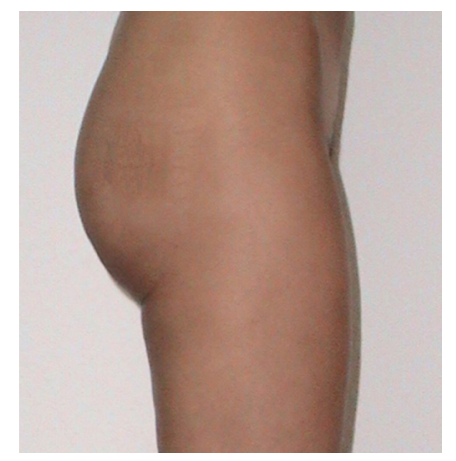

Fig. (5). Buttock lift in a young patient. A. Before, B. After. Young patients are mostly candidates for beautification and mini-invasive procedures. 
occurred we changed the suture and now we use the Bulgarian antimicrobial one having a short elasticity. In the second case the Streptococcus local infection was easily treated.

(A)
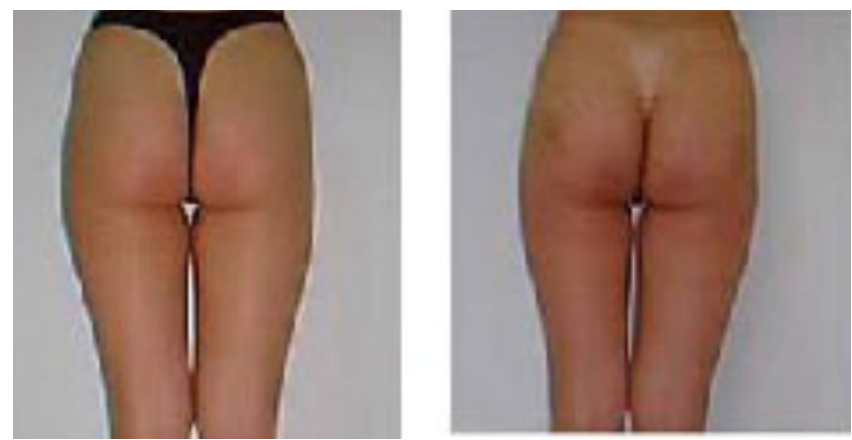

(B)
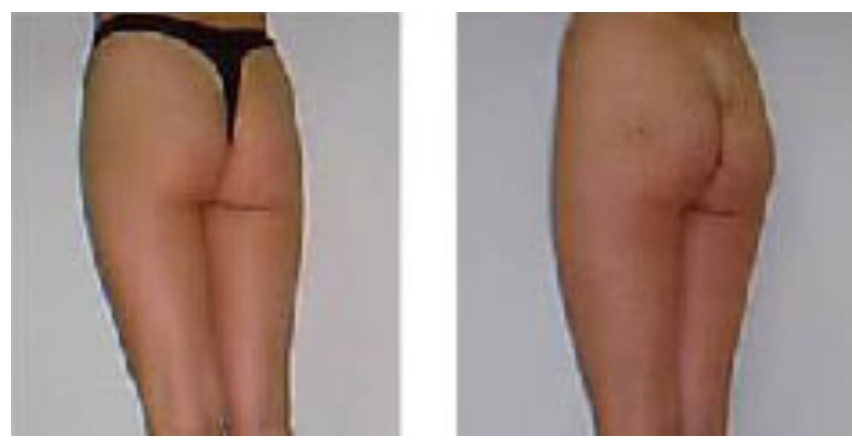

(C)
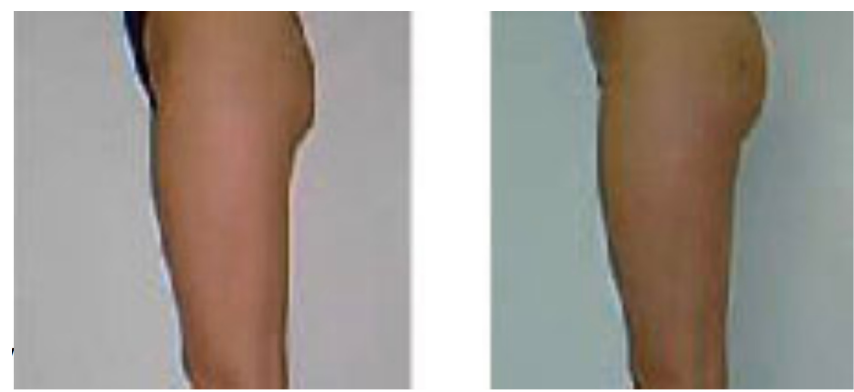

Fig. (6). Results after a buttock lift by suture only; A. Back view: before and after; B. Halfprofile: before and after; C. Profile view: before and after. Higher rounded buttock form is achieved. The only visible puncture scar of $1-2 \mathrm{~mm}$ could be visible in the lateral area of the buttock.

\section{DISCUSSION}

There is an increasing demand in modern society for surgical correction of the body contour. There are a limited number of operations specifically indicated for correction of non-aesthetic buttocks form, as a part of the totality of body appearance and proportion esthetics. The hips, thighs and the lower back frame the buttock contours. Buttock proportions are balanced by the anterior projection of the breasts. Ethnic differences in the shape and proportions of the buttocks create a variety of aesthetic variations in size and shape.

Flat and sagging buttocks without fat deposits are a common clinical condition, but there were no proven aesthetic and effective therapeutic options for buttocks lift and projection, previously to our technique.
(A)

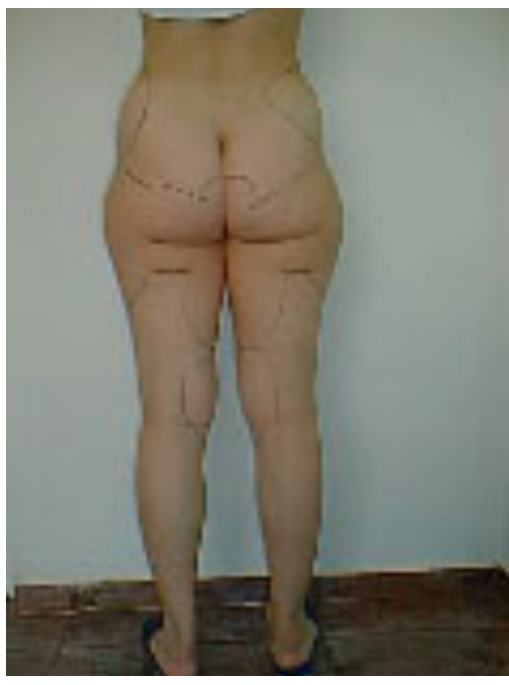

(B)

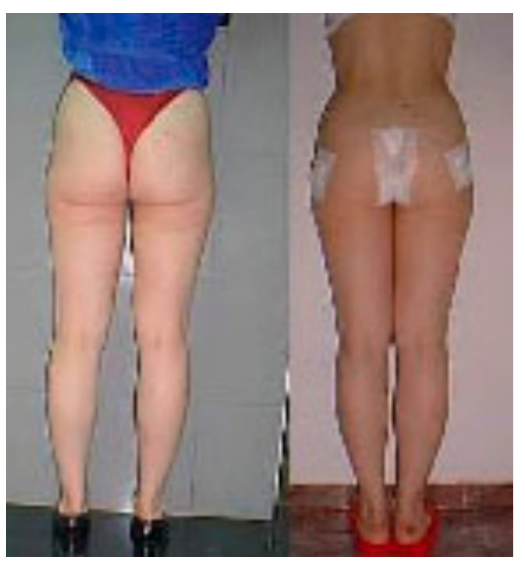

(C)

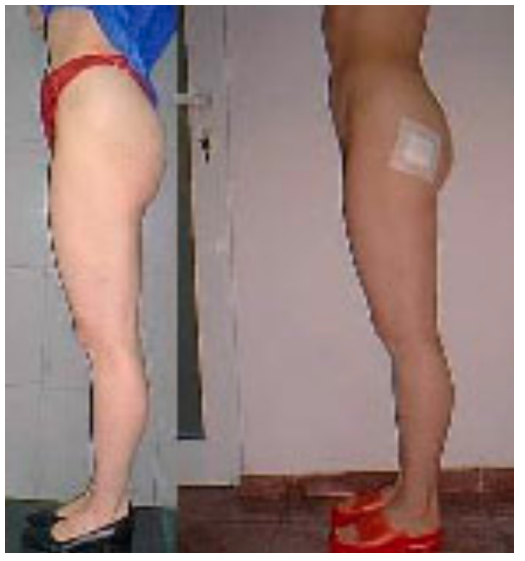

Fig. (7). A. The patient before total UAL of body and lower extremities. UAL was performed two months before the buttock lift by suture for total body and leg beautification as well as to reduce the heaviness of the hanging buttock soft tissue. Back view of the same patient before UAL of body and extremities; B. Buttock lift by suture: result on day one after surgery (back view): Buttocks are softly lifted, the subgluteal is raised and shortened; C. result on day first after surgery (profile view). "Happier" buttock form and elongation of the legs is visible. The closeness of the wounds to the anus area makes antibiotic prophylaxis and strict hygiene obligatory. 
(A)

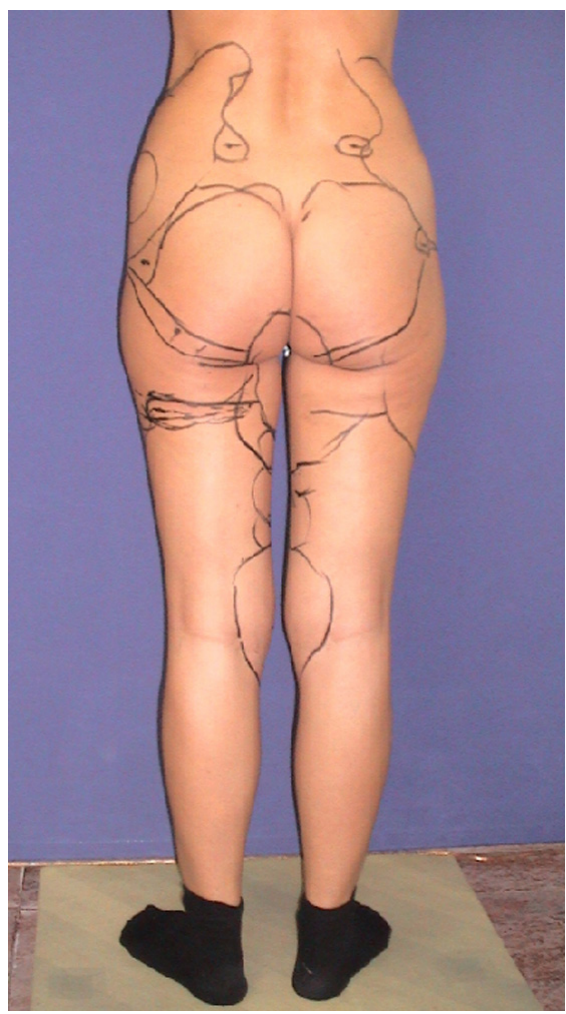

(B)

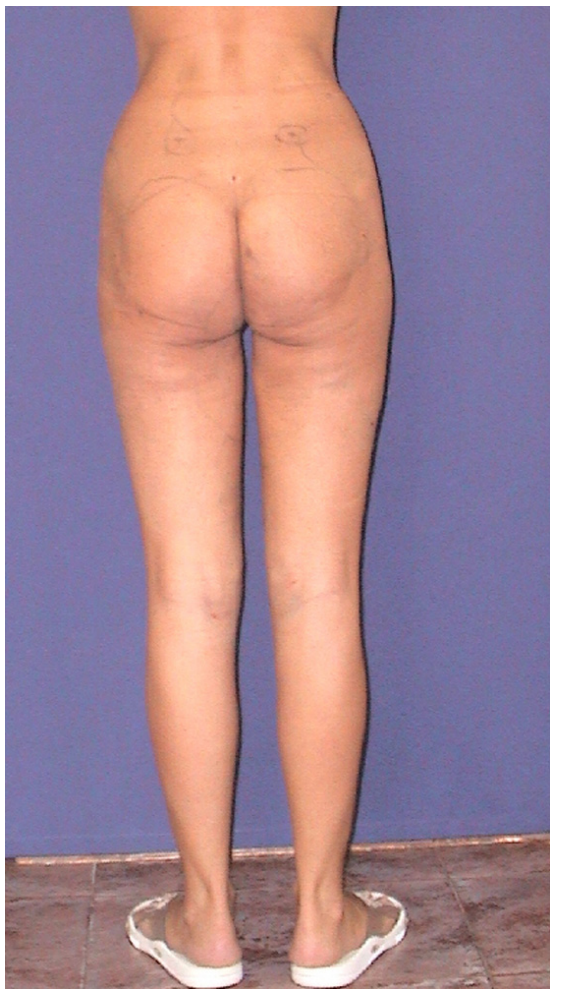

Fig. (8). A. Before, B. After a buttock lift combined with UAL lower limbs and trunk beautification. Result on day one. Drawings are still visible. Proportions are changed due to higher position of the buttocks. "Knee lifting" by UAL - creation of straight and elongated legs is one of the specialties of the author. Buttocks are lifted, infragluteal fold is lifted by $4-5 \mathrm{~cm}$ visibly elongating the lower limbs.
(A)

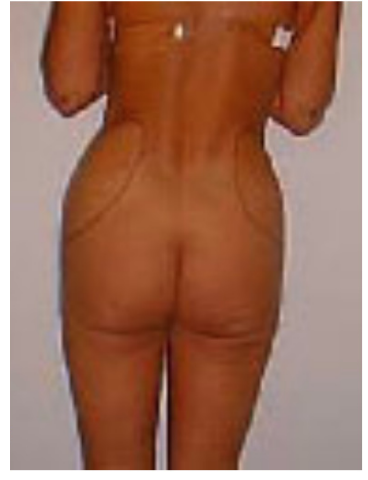

(B)

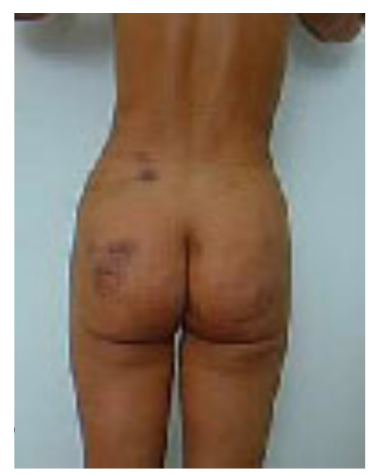

(C)

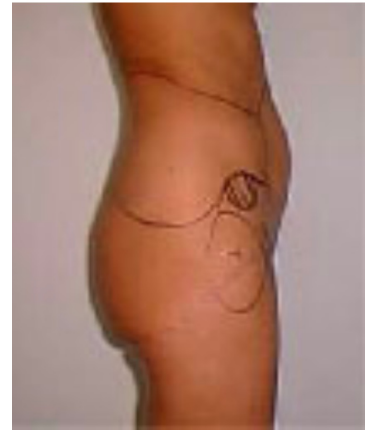

(D)

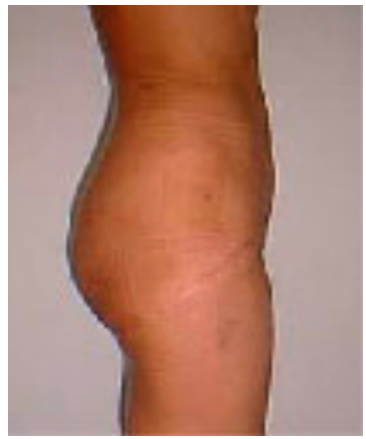

Fig. (9). A, C. A patient that was treated previously by excision lipoplasty of the inner, lateral thigh and buttocks (visible scars, irregularities and deformities). B, D. Result one day after buttock lift by suture and additional simultaneous UAL of flanks and abdomen. The buttocks are visibly lifted and a better-rounded form obtained. The use of UAL assured a body form beautification and at the same time a correction of irregularities and deformities. 
Subcision is a surgical technique that is used in treating advanced degree cellulite [11]. To treat excesses of fat and skin tissue in that area, liposuction [12-14] and/or dermolipectomy $[15,16]$ are mostly used. Liposuction is performed through small skin incisions, which results in minimal scar formation and is associated with minimal complication rates. The indication for liposuction is restricted to the conditions in which the overlying skin is capable of retracting and adapting itself to the new contour. Otherwise, if excess skin is the cause of the deformity, a dermolipectomy is mostly performed. In these cases, the incisions have to be chosen in a way that the resulting scar may be hidden as well as possible.

Liposuction of the buttock area is less mentioned in the literature and for some authors it is a forbidden zone [17]. Two additional approaches in suction lipectomy of the buttock region are described: liposuction of the "banana" and liposuction of the "sensuous triangle" [13]. The banana is the highest part of the posterior thigh just below the buttock crease. It appears only in certain individuals and appears as a bulge. Controlled scar retraction of the thin cutaneous adipose flap allows for good results even in flaccid and aged skin due to some authors $[14,18]$. A common complication of liposuction of this area is ptosis of the buttock crease The sensuous triangle is at the junction of the lateral buttock, lateral thigh, and posterior thigh. The use of ultrasound to improve the liposuction possibilities in body contouring [19, 20].

To improve buttock roundness and projection, fat transplantation and different implants, including mammary ones, were introduced $[3,21,22]$. An augmentation to sagging or hypertrophic buttocks similar to that of the breasts can be done [23].

Free fat graft has been used with success in cosmetic surgery to avoid the most common complications of doing a buttock augmentation with silicone prostheses and to find a better surgical procedure that is simpler, complementary with liposuction, and better able to deal with subtle body irregularities. In trying to achieve symmetry and better contour of the back torso and middle third of the body, the combination of liposuction and lipoinjection is rapidly becoming the procedure of choice for most of authors. Fat grafting is done in multiple tunnels in a deep plane [24], results are considered uniformly satisfactory. Liposuction is done with a tumescent technique in the lumbosacral, trochanteric, and subgluteal region to improve gluteal shape using additional lipofilling. Contour defects treated with autologus intramuscular fat graft injections need overcorrection by approximately 50 percent more volume. It is not an easy goal because of the high reabsortion of fatty tissue [25]. Delicate tissue handling and small total amount of fat transplanted by careful distribution in the recipient tissues are probably the factors responsible for longer lasting improvement in these patients [26]. Complications are minimal with enhanced satisfaction of both patients and surgeons.

In obese patients the functional benefits of a combination excision-suction lipectomy outweigh the disadvantages of the scarring [27]. If there is considerable excess of skin and tissue, excision procedures are performed to remove excess tissue by surgical resection via appropriately large incisions.
Resulting scars from excision surgery are always visible (Fig. 9C, D). Lipectomy with suction of the lower extremities has been of greater interest in recent years. Due to some authors the number of patients seeking dermolipectomy of the trunk and thighs is increasing. The so-called "lower body lift" combines the transverse flank/thigh/buttock lift and the fascial anchoring medial thigh lift in one operation [29]. Secondary high-buttock corrections pose difficult problems because of the poor vascularization of certain areas, the limited mobilization of the soft tissue, and the tendency toward poor scar formation. These factors limit the surgical techniques available. The tendency for the deformity to recur may necessitate several corrective procedures [4]. The deep planed torsoabdominoplasty is beneficial for treating gestation sequelae of the torso-abdominal wall, ptosis of the abdomen, vertical and horizontal enlargements of the musculoaponeurotic system, lipodystrophy, stretch marks, rhytidosis of the inguinal region, and ptosis of the external quadrant of the gluteus and the external trochanter area in one surgical procedure [30]. It creates pexy of the external quadrant of the gluteus region. Muscle strength is the limiting factor in repetitive squat lifting. Fatigue may be one of the determinants for changes in kinematics and choice of technique in lifting tasks. Lower body lift with superficial fascial system suspension is introduced to treat laxity of the entire lower trunk and thigh regions in one stage in selected patients This procedure needs 3 weeks off work and is expected to result in a tightening of the flank, buttocks, and total thighs. Minor complications are significantly higher than with the component procedures alone and occur in nearly 50 percent of patients [29]. Another surgical procedure, a circumferential torso excision was designed and utilized for minimal number of patients. This technique dramatically reduces the lateral flank and posterior tissue rolls to improve the operative results. Contour improvement of the buttocks and lateral thighs is produced as well [31]. A buried dermal-fat flap technique is particularly applicable to patients with asymmetry of the buttocks and thighs as well as those with ptosis of the buttocks. An advantage is that it creates a new gluteal fold at a predetermined higher level [16]. Belt lipectomy includes the traditional abdominoplasty or panniculectomy with excision extended laterally around the entire trunk. This technique yields a lateral thigh and buttock lift, and when combined with liposuction is used to improve contour of the thighs [32].

Correction of sequelae of primary hip-buttock-thigh plasty has become a common challenge in aesthetic plastic surgery. Due to some authors, suggested techniques for dealing with this problem include denuding the skin at the depressed area, pulling flaps upward and outward, using dermal buried flaps, and utilizing liposuction. Liposuction can be used successfully in combination with classic hipbuttock-thigh plasty to enhance the aesthetic result as well as to facilitate the surgical technique [28].

Excision body lifts are surgical procedures that are infrequently performed because the length of operating time increases the risk to the patient as well as the likelihood of surgeon fatigue. The other drawback of body lifts is the long incision line. However, these incisions are well accepted if they are well placed and if the results of body change are significant. Meticulous haemostasis, limited undermining, 
and the closure of dead space are factors that produce a more reliable procedure, both in terms of postoperative problems and the final results [33]. The transgluteal approach could be responsible, according to some authors for bad clinical results, due to injury of the nervus gluteus superius. Many anatomical variations are found concerning the point of the nerve's division into 2 branches, nearer or farther from the foramen ischiadicum [34].

\section{CONCLUSION}

Redundant tissue in sagging buttocks can be corrected by excision lifts. However, these are seldom used procedures because of postoperative problems such as unaccepted inferiorly displaced and wide scars, and early recurrence of ptosis, large trauma and blood loss, prolonged post op period.

In order to limit these complications in flat and sagging buttocks without remarkable fat deposits, we developed a surgical technique using a circumferential suture of the buttock's soft tissue. The fascial suspension gives strong vertical support with minimal tension on the skin, and reduces the complications traditionally associated with such procedures. The results of our operations are aesthetically compatible with and even much better than non-scarring techniques such as UAL and liposuction solely in young patient having strong and elastic skin and tissue, described in our presentations as well $[19,35]$. Liposuction alone can not lift. The author's operation offers fewer complications than any other described.

In patients, whose problem was excessive fat in conjunction with skin flabbiness, UAL of the buttocks and surrounding tissues for beautification body contouring, combined with the buttock lift suture method, completed the main goals of the procedure.

Buttocks lift is an efficient and safe procedure to correct or enhance buttock contour. It has virtually eliminated blood transfusions and the major complications of dermolipectomies and liposuction under general anaesthesia.

The author's surgical procedure using a suture is simple, atraumatic and low in cost, with minimal morbidity and very good results. It is important to note that a good result does not depend on great surgery but rather on more simple, acceptable procedures for patients, resulting in harmonious structuring and positioning of the form, lifting of the lower portion of the buttocks, augmentation in the upper gluteus and better projection. high.

Complications are very few, and patient satisfaction is

\section{CONFLICT OF INTEREST}

The author confirms that this article content has no conflicts of interest.

\section{ACKNOWLEDGEMENT}

Declared none.

\section{REFFERENCES}

[1] Serdev NP. Suture suspensions for lifting or volume augmentation in face and body. 2nd Annual Meeting of the National Bulgarian Society for Aesthetic Surgery and Aesthetic Medicine; 1994 March $18^{\text {th }}$, Sofia, 1994; pp. 11-8.
[2] Serdev NP. Year 1994, Suture suspension for lifting or volume augmentation in face and body (English version). Int $\mathrm{J}$ Cosmet Surg 2001; 1(1): 2561-8.

[3] Cardenas-Camarena L, Lacouture AM, Tobar-Losada A. Combined gluteoplasty: liposuction and lipoinjection. Plast Reconstr Surg 1999; 104 (5): 1524-31.

[4] Regnault P, Daniel R. Secondary thigh-buttock deformities after classical techniques. Prevention and treatment. Clin Plast Surg 1984; 11(3): 505-16.

[5] Karnes J, Salisbury M, Schaeferle M, Beckham P, Ersek RA. Hip lift. Aesthetic Plast Surg 2002; 26(2): 126-9.

[6] Hagen K, Sorhagen O, Harms-Ringdahl K., Influence of weight and frequency on thigh and lower-trunk motion during repetitive lifting employing stoop and squat techniques. Clin Biomech (Bristol, Avon) 1995; 10(3): 122-7.

[7] Niechajev I, Sevcuk O. Long-term results of fat transplantation: clinical and histologic studies. Plast Reconstr Surg 1994; 94(3): 496-506.

[8] Guerrerosantos J. Autologous fat grafting for body contouring. Clin Plast Surg 1996; 23(4): 619-31.

[9] Peren PA, Gomez JB, Guerrerosantos J, Salazar CA. Gluteus augmentation with fat grafting. Aesthetic Plast Surg 2000; 24(6): 412-7.

[10] Ichida M, Kamiishi H, Shioya N. Aesthetic surgery of the trunk and extremities in the Japanese. Ann Plast Surg 1980 Jul; 5(1): 31-9.

[11] Hexsel DM, Mazzuco R., Subcision: a treatment for cellulite. Int J Dermatol 2000; 39(7): 539-44.

[12] Gargan TJ, Courtiss EH. The risks of suction lipectomy. Their prevention and treatment. Clin Plast Surg 1984; 11(3): 457-63.

[13] Schlesinger SL. Two arcane areas in liposuction: the banana and the sensuous triangle. Aesthetic Plast Surg 1991; 15(2): 175-80.

[14] Gasparotti M. Superficial liposuction: a new application of the technique for aged and flaccid skin. Aesthetic Plast Surg 1992; 16(2): 141-53.

[15] Pitanguy I. Surgical reduction of the abdomen, thigh, and buttocks. Surg Clin North Am 1971; 51(2): 479-89.

[16] Delerm A, Cirotteau Y. Cruro-femoro-gluteal or circumgluteal plasty. Ann Chir Plast 1973; 18(1): 31-6.

[17] Shaer WD. Gluteal and thigh reduction: reclassification, critical review, and improved technique for primary correction. Aesthetic Plast Surg 1984; 8(3): 165-72.

[18] Gasperoni C, Salgarello M. MALL liposuction: the natural evolution of subdermal superficial liposuction. Aesthetic Plast Surg 1994; 18(3): 253-7.

[19] Serdev NP. Buttock lift. Two own methods. 3rd International Congress of the South-American Academy of Cosmetic Surgery, Buenos Aires, Argentina, 2001; pp. 37-8.

[20] Serdev NP. Buttocks lift by ultrasonic assisted liposuction - My technique. Int J Aesthet Cosmet Beauty Surg 1991; 1(3): 130-54.

[21] Chajchir A. Fat injection: long-term follow-Up. Aesthetic Plast Surg 1996; 20(4): 291-6.

[22] Lack EB. Contouring the female buttocks. Liposculpting the buttocks. Dermatol Clin 1999; 17(4): 815-22.

[23] Lewis JR Jr. Body contouring. South Med J 1980; 73(8): 1006-11.

[24] Pereira LH, Radwanski HN. Fat grafting of the buttocks and lower limbs. Aesthetic Plast Surg 1996; 20(5): 409-16.

[25] Hanke CW, Bullock S, Bernstein G., Current status of tumescent liposuction in the United States. National survey results. Dermatol Surg 1996; 22(7): 595-8.

[26] de Pedroza LV. Fat transplantation to the buttocks and legs for aesthetic enhancement or correction of deformities: long-term results of large volumes of fat transplant. Dermatol Surg 2000; 26(12): 1145-9.

[27] Teimourian B, Adham MN. Anterior periosteal dermal suspension with suction curettage for lateral thigh lipectomy. Aesthetic Plast Surg 1982; 6(4): 207-9.

[28] (a) Guerrerosantos J. Secondary hip-buttock-thigh plasty. Clin Plast Surg 1984; 11(3): 491-503. (b) Lewis JR Jr. Body contouring. South Med J 1980; 73(8): 1006-11.

[29] Lockwood T. Lower body lift with superficial fascial system suspension. Plast Reconstr Surg 1993; 92(6): 1112-22.

[30] Gonzalez M, Guerrerosantos J. Deep planed torso-abdominoplasty combined with buttocks pexy. Aesthetic Plast Surg 1997; 21(4): 245-53.

[31] Carwell GR, Horton CE Sr. Circumferential torsoplasty. Ann Plast Surg 1997; 38(3): 213-6. 
[32] Heddens CJ. Belt lipectomy: procedure and outcomes. Plast Surg Nurs 2001; 21(4): 185-9, 199.

[33] Pascal JF, Le Louarn C. Remodeling bodylift with high lateral tension. Aesthetic Plast Surg 2002; 26(3): 223-30.

[34] Lavigne P, Loriot de Rouvray TH. The superior gluteal nerve. Anatomical study of its extrapelvic portion and surgical resolution by trans-gluteal approach Rev Chir Orthop Reparatrice Appar Mot 1994; 80(3): 188-95.

[35] Lawrence N, Coleman WP 3rd. The biologic basis of ultrasonic liposuction. Dermatol Surg 1997; 23(12): 1197-200.

(C) Nikolay P. Serdev; Licensee Bentham Open.

This is an open access article licensed under the terms of the Creative Commons Attribution Non-Commercial License (http: //creativecommons.org/licenses/by$\mathrm{nc} / 3.0 /$ ) which permits unrestricted, non-commercial use, distribution and reproduction in any medium, provided the work is properly cited. 